\title{
4 INTEGRATION VON GEFLÜCHTETEN IM LÄNDLICHEN RAUM
}

Empirische Forschungen zur Integration von Geflüchteten haben sich traditionell eher mit dem urbanen Raum beschäftigt. Zur Behandlung dieser Thematik in ruralen Kontexten existieren vergleichsweise wenige wissenschaftliche Studien. Obwohl der ländliche Raum tendenziell eher als Abwanderungsgebiet denn als Ziel für Wanderungsbewegungen angesehen wird (REITTER 2017, p. 36), nimmt die Bedeutung internationaler Migration in diese Gebiete in den letzten Jahren erheblich zu (MACHOLD \& DAX 2016, p. 63).

Das Gegensatzpaar Stadt-Land hatte in der Migrationsforschung insofern großen Einfluss, da die Binnenwanderung aus den ländlichen Regionen in den städtischen Bereich hier viel Raum einnimmt (MARKom 2009, p. 37). Die Rollen sind in dieser dichotomen Vereinfachung klar verteilt: Aus unterschiedlichen Gründen zieht es viele Menschen aus abgelegenen Gegenden in die Städte, wo sie sich bezüglich Arbeitsmöglichkeiten, kulturellen Angeboten oder Infrastruktur bessere Bedingungen erwarten. Gerade aufgrund dieser Abwanderung oder „Landflucht“ ist internationale Migration aber für viele Gemeinden ein interessantes Thema geworden. Damit kann beispielsweise geringen Geburtenraten und einer rückläufigen Bevölkerungsentwicklung entgegengewirkt werden, womit die lokale Infrastruktur wie Schulen und Geschäfte erhalten bleiben (GRIESSER 2017). Migration und die Aufnahme von Geflüchteten stellt Gemeinden somit nicht nur vor Herausforderungen, sondern bietet auch eine Reihe von Chancen, die auf Basis entsprechender Strategien genutzt werden können.

Der ländliche Raum wird häufig mit Attributen wie Nähe, gegenseitigem Vertrauen und Solidarität verbunden, aber gelegentlich auch als „ländliche Idylle“ verklärt, in welcher Menschen ein ursprüngliches Leben im Einklang mit der Natur führen (MACHOLD \& DAX 2016, p. 64). Die damit einhergehenden Vorstellungen reichen von einer Dominanz der Landwirtschaft als bedeutendster Subsistenzform über hohe Abwanderungsraten und starken Bevölkerungsrückgang bis zur Unattraktivität für Wirtschaft und Industrie (ebd.). Diese Stereotypen treffen allerdings nur auf einige wenige Gegenden zu, genauso wenig kann die strikte Aufteilung in Stadt und Land in dieser Form aufrechterhalten werden (ReITTER 2017, p. 15). Außerdem kann der ländliche Raum sehr unterschiedlich strukturiert sein: je nachdem ob sich in der Nähe beispielsweise größere Städte oder Tourismusregionen befinden. MACHOLD und DAx (2016) sprechen daher auch von der „Diversität ländlicher Räume“. Diese Diversität spiegelt sich ebenfalls in den unterschiedlichen Unterbringungssituationen von Asylwerber/inne/n in österreichischen Gemeinden und den damit verbundenen Möglichkeiten und Einschränkungen wider.

In Österreich werden Asylwerber/innen über ein Quotensystem nach der Registrierung in den Erstaufnahmezentren Traiskirchen (NÖ) oder Thalham (OÖ) auf die Bundesländer und Gemeinden verteilt. Ziel dieses Ansatzes ist es, eine dezentrale Unterbringung von Geflüchteten zu fördern (Weidinger, Kordel \& PoHLE 2017, p. 
48), was in manchen Fällen aber dazu führt, dass Quartiere an sehr abgelegenen Orten eingerichtet werden, in denen die Asylwerber/innen dann faktisch von der Außenwelt abgeschnitten sind. Begründet wird dieses Vorgehen einerseits mit einer Lastenteilung zugunsten der größeren Städte, in denen die Situation auf dem Arbeits- und Wohnungsmarkt oft ohnehin schon angespannt ist (ebd.). Auf der anderen Seite wird die,,räumliche Nähe zu Infrastrukturen und unterstützenden Akteuren“" (ebd.) in ländlichen Gebieten als Chance zur Integration von Geflüchteten gesehen. In vielen Fällen ist die Unterbringung von Asylwerber/inne/n in den Gemeinden nur vorübergehend, da die meisten nach dem Abschluss des Asylverfahrens in größere Städte ziehen (siehe Abschnitt 7.5.2). Diejenigen, die aber vor Ort wohnhaft bleiben, können tatsächlich einen wichtigen Beitrag dazu leisten, den Bevölkerungsrückgang in ländlichen Regionen zu verringern. Im Folgenden werden die strukturellen Rahmenbedingungen näher beschrieben, welche in jenen Gemeinden vorhanden waren, in denen die dieser Publikation zugrundeliegenden Daten gesammelt wurden.

\subsection{Marktgemeinde St. Andrä-Wördern}

Die Marktgemeinde St. Andrä-Wördern liegt im Osten des Bezirks Tulln in Niederösterreich und umfasst neben St. Andrä und Wördern fünf weitere Katastralgemeinden. Inklusive der Nebenwohnsitze ergibt sich eine Gesamtbevölkerung von rund 10.000 Personen (Gemeinde St. Andrä-Wördern o.J.). Laut Angaben der Statistik Austria für das Jahr 2018 ist die Bevölkerung von St. Andrä-Wördern seit dem Jahr 2001 um 22\% gewachsen ${ }^{7}$. Dieser Zuzug ist vor allem auf die Lage im Umland von Wien zurückzuführen, die viele Menschen anspricht, welche im Grünen wohnen, aber trotzdem die Vorzüge der nahen Großstadt genießen wollen.

Die „Urban-Rural-Typologie“ der Statistik Austria weist St. Andrä-Wördern als „ländlichen Raum im Umland von Zentren“ aus (Statistik Austria 2018). Mit dieser Kategorie werden jene Gebiete bezeichnet, die einen gewissen Anteil an Pendler/inne/n aufweisen und im Umland von größeren Städten liegen. Innerhalb der Zuordnung „Umland von Zentren“ wird St. Andrä-Wördern als zentral eingestuft, da die nächste größere Stadt, in diesem Fall Wien, durch motorisierten Individualverkehr innerhalb von 30 Minuten erreicht werden kann (STATISTIK Austria 2016). Was die Verkehrsanbindung betrifft, besteht von der Gemeinde aus eine gute öffentliche Zugverbindung nach Wien. An der S-Bahnlinie 40 gelegen, welche ausgehend vom Franz-Josefs-Bahnhof Wien mit Tulln beziehungsweise St. Pölten verbindet, befinden sich die Bahnhöfe GreifensteinAltenberg sowie St. Andrä-Wördern unmittelbar auf dem Gemeindegebiet. Tagsüber gibt es hier alle 30 Minuten eine Verbindung zwischen Wien und Tulln, sodass Pendelverkehr auch auf der Schiene möglich ist. St. Andrä-Wördern wird in dieser „UrbanRural-Typologie“" also dem ländlichen Raum zugeordnet, allerdings innerhalb desselben

$7 \quad$ Ausgegangen wurde dabei von einer Bevölkerungszahl von 7.870 Personen für das Jahr 2018 (vgl. StatistiK Austria 2018). 
im Einzugsgebiet einer größeren Stadt verortet. Hinsichtlich der oben angesprochenen Diversität ländlicher Räume bedeutet dies, dass die Gemeinde durch diese Lage andere Möglichkeiten bietet als viele Gemeinden des Most- und Waldviertels. In der Prognose der regionalen Bevölkerungsentwicklung für Niederösterreich weist die Region um Tulln ein erwartetes Wachstum von 17\% bis zum Jahr 2040 auf und liegt damit weit über dem Schnitt von 8,4\% für Niederösterreich insgesamt (ÖSTERREICHISCHE RAUMORDNUNGSKONFERENZ 2019). Obwohl das genannte Motiv des Erhalts von Infrastruktur durch die Ansiedlung von Geflüchteten in St. Andrä-Wördern keine Rolle spielt, werden vom zuständigen Gemeinderat für Migration und Integration positive Aspekte der Anwesenheit von Geflüchteten genannt. So werde beispielsweise der soziale Zusammenhalt in der Gemeinde gestärkt, da sich die Zivilgesellschaft formiert und zeigt, was sie zu leisten bereit ist (Interview GEMEINDERAT Für SOZIAL- GeSUNDHEITSWESEN: Integration, Migration, Freizeit, Veranstaltungen St. Andrä-Wördern 2018).

Im Gegensatz zu vielen anderen Gemeinden des ländlichen Raumes in Österreich sind die Rahmenbedingungen in St. Andrä-Wördern nicht durch Abwanderung und Landflucht, sondern durch eine positive Bevölkerungsentwicklung bestimmt. Infolge dieses Bevölkerungswachstums ist es nicht einfach, den notwendigen Wohnraum zur Verfügung zu stellen. Dies beeinflusst sowohl die Möglichkeit von Asylwerber/inne/n während des Verfahrens in privaten Unterkünften zu wohnen als auch die Chancen von Asylberechtigten, sich nach dessen positivem Abschluss in der Gemeinde niederzulassen. Ohne die Unterstützung von engagierten Ehrenamtlichen ist dies in beiden Fällen kaum möglich. Internationale Migration spielt allerdings im Rahmen der Bevölkerungszunahme der letzten Jahre nur eine geringe Rolle: im Oktober 2016 waren knapp über 100 Asylwerber/innen in der Gemeinde untergebracht (HaGENTHALER 2016). Diese waren Großteiles Erwachsene, die deutliche Mehrheit davon Männer. Es waren zu diesem Zeitraum aber auch rund zehn unbegleitete minderjährige Geflüchtete und mehrere Familien in St. Andrä-Wördern wohnhaft. Die Herkunftsländer der Geflüchteten waren vielfältig, wobei viele aus Afghanistan, Syrien, dem Iran, dem Irak und Somalia kamen.

In der Gemeinde St. Andrä-Wördern wurden bereits seit 1956 im Haus der Österreichischen Jungarbeiterbewegung (ÖJAB) ${ }^{8}$ immer wieder Geflüchtete untergebracht. Dieses interkulturelle Wohnheim in Greifenstein beherbergte seit 2001 bis zu 65 volljährige und überwiegend männliche Asylwerber/innen aus circa 20 verschiedenen Nationen. Die Anzahl der Bewohner/innen war über die Jahre Schwankungen unterworfen. So wohnten dort bis zur vorübergehenden Schließung Ende Juni 2018 noch knapp unter 50 Asylwerber/innen in Zimmern mit bis zu vier Personen. Nach

$8 \quad$ Die ÖJAB wurde kurz nach dem Zweiten Weltkrieg gegründet, um den Arbeiter/inne/n, die am Wiederaufbau des Stephansdomes nach Kriegsende arbeiteten, eine Unterkunft zu bieten (Österreichische JungarbeiterBEwEGUNG o.J.). Im Herbst 2018 umfasste das Angebot der ÖJAB unter anderem den Betrieb von Studierenden- und Senior/inn/en/heimen in mehreren Bundesländern sowie die Unterbringung von unbegleiteten minderjährigen Geflüchteten an zwei Standorten in Niederösterreich. 
einer Renovierung im Sommer wurde das ÖJAB-Haus als Unterkunft für bis zu 48 unbegleitete minderjährige Flüchtlinge im Herbst wiedereröffnet. Schließlich folgte die Beendigung der Unterbringung von Geflüchteten an diesem Standort mit Ende Mai 2020, da ein entsprechender Bedarf an Betreuungsplätzen nicht mehr gegeben war.

Aufgrund der geografischen Lage der Unterkunft als östlichstes Haus in der östlichsten Katastrale der Gemeinde wurde das ÖJAB-Haus in St. Andrä-Wördern zeitweise nur wenig wahrgenommen. Der nächstgelegene Bahnhof war jener von Höflein und lag damit nicht mehr im Gemeindegebiet. Dadurch hatten viele Bewohner/ innen eher Bezug zu Wien beziehungsweise Klosterneuburg als zu St. Andrä-Wördern (Interview Gemeinderat Für Sozial- Gesundheitswesen: InTEGration, Migration, Freizeit, Veranstaltungen St. Andrä-Wördern 2018). Die Unterkunft wurde alle zwei Wochen von der mobilen Beratung der Caritas in Korneuburg besucht, auch auf das in Wien zur Verfügung stehende Angebot in diesem Bereich wurde von der Heimleitung oder Ehrenamtlichen immer wieder verwiesen. Die gute öffentliche Anbindung an Wien ermöglichte es vielen der Asylwerber/inne/n trotz der ruhigen Lage nicht unter der typischen Abgeschiedenheit zu leiden, welche viele andere Unterkünfte für Geflüchtete in ländlichen Regionen charakterisiert. Generell wurden die Bedingungen für die Unterbringung von Asylwerber/innen im ÖJAB-Haus in Greifenstein immer wieder als sehr gut beschrieben (vgl. UNHCR WIEN 2013). Neben den angebotenen Möglichkeiten zur Freizeitgestaltung, die unter anderem einen Gemeinschaftsgarten und einen Fußballplatz umfassten, ist hier insbesondere das Engagement von Ehrenamtlichen zu nennen. Diese setzten verschiedene Freizeitaktivitäten um und organisierten einen Deutschkurs für die Bewohner/innen.

Federführend dabei war vor allem der Verein „Grenzenlos St. Andrä-Wördern“, der im Jahr 2004 gegründet wurde. Ausgehend von einer Gruppe von Personen, die einander trafen, um gemeinsam zu kochen und zu essen, hat sich die Palette des Angebotes im Laufe der Jahre deutlich verbreitert. So gibt es inzwischen verschiedene Untergruppen, welche sich zum Teil überschneiden, zum Teil aber auch recht unabhängig voneinander agieren. Dazu zählen neben den Kochabenden unter anderem Fußballtraining, Tanzabende, Weltcafés aber auch Literaturveranstaltungen. Die Idee von „Grenzenlos““ ist es, die Dorfgemeinschaft zusammenzubringen und einen Raum für gemeinsame Aktivitäten und Austausch zu schaffen. Dabei soll insbesondere die im Ort vorhandene Vielfalt sichtbar gemacht und als Bereicherung verstanden werden.

Einen weiteren wichtigen Beitrag zur Integration von Geflüchteten leistet der „Grenzenlos“ Solidarfonds. Über diesen Fonds werden für Geflüchtete, welche in Wien oder Tulln Deutschkurse besuchen, Monatskarten für die öffentlichen Verkehrsmittel finanziert, da Asylwerber/innen diese meist nicht selbst bezahlen können. Außerdem können Unterlagen für die Kurse gekauft und in manchen Fällen auch Prüfungsgebühren übernommen werden 9 . Damit werden auf der einen Seite Einschränkungen

$9 \quad$ Finanziert werden diese Aktivitäten durch viele kleinere Spender/innen und manche größere Beiträge, die beispielsweise anlässlich von Geburtstagen anstelle von Geschenken gesammelt wurden (Interview LeITER „GRENZENLOS“ SOLIDARFONDS 2018). 
in der Mobilität von Asylwerber/inne/n aufgrund geringer eigener finanzieller Mittel überwunden und andererseits Möglichkeiten zur Verbesserung der Sprachkenntnisse eröffnet, die über das eigene Angebot in der Gemeinde hinausgehen. Dies wirkt sich positiv auf die Möglichkeiten von Geflüchteten aus, ihren Alltag selbstbestimmt zu gestalten und trägt damit auch dazu bei, die Verwirklichungschancen von Asylwerber/ inne/n zu erhöhen.

Als in den Jahren 2015 und 2016 in Österreich viele Geflüchtete ankamen, waren in der Gemeinde bereits langjährige Erfahrungen mit Asylwerber/inne/n und einige Ehrenamtliche vorhanden, die sich im Verein „Grenzenlos“ für diese engagierten. Infolge der großen Fluchtbewegungen in dieser Zeit wurden in St. Andrä-Wördern zwei weitere zivilgesellschaftliche Initiativen zur Unterstützung von Geflüchteten gegründet. Die beiden Gruppierungen nannten sich „Das Dorf hilft“ und „St. Andrä-Wördern hilft“. Während sich die Initiative „Das Dorf hilft“ vor allem um Sachspenden bemühte und versuchte, Gegenstände des täglichen Gebrauchs für die Neuankömmlinge bereitzustellen, bezog sich die Unterstützung von „St. Andrä-Wördern hilft“ vorwiegend auf die organisatorischen Abläufe nach der Ankunft. So wurden Geflüchtete bei Amtswegen oder zu Arztbesuchen begleitet, bei der Anmeldung von Kindern in der Schule und im Kindergarten unterstützt und durch das Angebot eines Deutschkurses erste Möglichkeiten zur Verständigung geschaffen. Zusätzlich wurden für Familien, aber auch für einzelne Asylwerber/innen, private Wohnungen vermittelt, in denen diese zum Teil zu sehr günstigen Konditionen wohnen konnten. Im Pfarrheim wurde eine zehnköpfige syrische Familie untergebracht und durch den Diakon tatkräftig unterstützt. Zwei weitere Familien fanden in einem Haus Unterkunft und Unterstützung, welches von einer in St. Andrä-Wördern ansässigen Familie vermietet wurde. Mit dem Rückgang der Anzahl an Asylwerber/inne/n verringerte sich auch das ehrenamtliche Engagement in diesem Bereich. So existiert die Initiative „Das Dorf hilft“ inzwischen nicht mehr. Was „St. Andrä-Wördern hilft" betrifft, sind einige der Ehrenamtlichen mittlerweile nicht mehr aktiv, andere beteiligen sich jedoch immer noch an den Aktivitäten von „Grenzenlos ${ }^{\text {“10 }}$.

Mit den Fluchtbewegungen von 2015 und 2016 begann auch eine intensive Debatte um die Unterbringung von weiteren Asylwerber/inne/n in St. Andrä-Wördern. Als im Herbst 2016 bekannt wurde, dass in der Dammstraße eine weitere Unterkunft für unbegleitete minderjährige Flüchtlinge eröffnet werden sollte, regte sich, vor allem seitens der unmittelbaren Anrainer/innen des geplanten Quartiers, Widerstand. Unter anderem wurden die unzureichende Informationspolitik im Vorfeld seitens des Landes Niederösterreich und des Betreibervereins „menschen.leben“ sowie die Wahl des Standortes kritisiert. In diesem Zusammenhang führte das jungen, männlichen Geflüchteten zugeschriebene Gefahrenpotential schließlich zur Durchführung einer Unterschriftenaktion gegen diese Unterkunft ${ }^{11}$. Die Initiator/inn/en der Unterschriftenaktion

$10 \mathrm{Ob}$ und in welcher Form der ehrenamtlich organisierte Deutschkurs in der Gemeinde St. Andrä-Wördern derzeit noch weitergeführt wird, ist den Autor/inn/en nicht bekannt.

11 Scheibelhofer (2017) betont, dass in diesem Zusammenhang vor allem die Kategorie „männliche muslimische Jugendliche“ von Bedeutung ist. Wegen des diesen Personen zugeschrie- 
sammelten knapp über 1.000 Unterschriften, welche dann dem Gemeinderat übergeben wurden (AmTSBlatt St. ANDRÄ-WÖRDERn 2016). Dennoch sprachen sich die Gemeinderät/inn/e/n mehrheitlich gegen die Umsetzung der Forderungen aus (GEMEINDE ST. ANDRÄ-WÖRDERN 2016).

Trotz der Kontroverse wurde die Unterkunft von zehn unbegleiteten minderjährigen Flüchtlingen bezogen, welche vom Verein „menschen.leben“ betreut wurden (AmtSblatt St. AndRÄ-Wördern 2016). In den Monaten zwischen September 2016 und April 2017 kam es dann aber doch immer wieder zu Schwierigkeiten mit den untergebrachten Jugendlichen ${ }^{12}$. Schlussendlich wurde im April als Reaktion darauf beschlossen, die Belegung zu ändern und anstatt der unbegleiteten Jugendlichen zogen eine Familie und neun erwachsene Männer in das Quartier in der Dammstraße (Amtsblatt St. AndRÄ-WÖRDERN 2017). Nachdem seit dieser Änderung keine Probleme auftraten, hat sich die Situation inzwischen wieder beruhigt. Der Betreiberverein „menschen.leben“ des Hauses in der Dammstraße wurde allerdings mit Ende des Jahres 2018 insolvent und das Quartier geschlossen. Mit der Unterstützung von Ehrenamtlichen konnten jedoch einige der Bewohner/innen in der Folge privat in der Gemeinde untergebracht werden.

\subsection{Erhebungsgemeinden im Mostviertel}

Im Mostviertel wurden drei Nachbargemeinden - im Text mit den Buchstaben A, B und C anonymisiert - als Erhebungsorte ausgewählt. Die drei Gemeinden sind mit dem Auto von St. Pölten in circa einer halben Stunde erreichbar. Darüber hinaus sind die Orte mittels einer Nebenbahn mit der Landeshauptstadt verbunden. Die Gemeinde A ist sowohl flächenmäßig als auch was die Bevölkerungszahl betrifft die größte Gemeinde. Sie liegt zwischen den beiden anderen Orten und ist in der Region gewissermaßen ein Zentrum mit breiteren Einkaufsmöglichkeiten und erweitertem Dienstleistungsangebot. Die Bevölkerungszahlen bewegen sich zwischen fast 2.000 (Gemeinde B) und mehr als 3.200 Einwohner/inne/n (Gemeinde A). Die Region ist nicht von Zuzug geprägt, wenngleich die Zahlen der Bevölkerungsstatistik Abwanderung in den Gemeinden nicht als triftiges Problem ausweisen. Lediglich für Gemeinde B, die am weitesten von der Landeshauptstadt entfernt liegt, zeigt sich mit Blick auf die letzten 20 Jahre, dass

benen Bedrohungspotenzials wurde auf unterschiedlichen Ebenen mit Abschottungsmaßnahmen reagiert. Einerseits erfolgte dies durch Grenzschließungen, um zu verhindern, dass weitere Geflüchtete nach Europa kommen. Selbiges wurde auch auf der Grundlage der mutmaßlichen Terrorgefahr argumentiert, die von dieser Gruppe ausgehen könnte. Andererseits wurden auf der Ebene von identitätspolitischen Grenzziehungen zunehmend exklusive Zugehörigkeitsmodelle propagiert, wie beispielsweise in der Debatte um die „Werte- und Orientierungskurse“ des Österreichischen Integrationsfonds deutlich wurde.

12 Einige der UMF litten an psychischen Problemen und es gab immer wieder Vorfälle, zu welchen die Polizei in das Quartier gerufen wurde (BeZIRKSBL ̈̈TTER 2017). Allerdings spielten sich die meisten dieser Ereignisse innerhalb der Unterkunft ab, sodass für die Gemeindebevölkerung keine wirkliche Gefahr bestand (Interview BÜRGERMEISTER ST. ANDRÄ-WÖRDERN 2018). 
die Bevölkerungszahlen leicht zurückgegangen sind (AMT DER NÖ LANDESREGIERUNG 2020).

Laut der „Urban-Rural-Typologie“ der Statistik Austria werden die drei Gemeinden dem ländlichen Raum zugeordnet (STATistiK Austria 2018). Die Gemeinde C, welche am nächsten an St. Pölten liegt, wird in dieser Klassifikation als zentraler ländlicher Raum eingestuft. Die beiden anderen Gemeinden als peripherer ländlicher Raum. Die Region ist vom Auspendeln geprägt. Der Anteil der Auspendler/innen beträgt um die $60 \%$ in den Gemeinden A und B, in der Gemeinde C liegt er sogar bei 70\% (AMT DER NÖ LANDESREGIERUNG 2020). Trotz der räumlichen Nähe sowie ähnlicher soziodemographischer Variablen (betreffend Wahlverhalten, Ausbildungsgrad, Wohnformen, Erwerbstätigkeit, Vereinsleben etc.) wiesen die drei Nachbargemeinden einen sehr unterschiedlichen Umgang mit Geflüchteten auf, der auch auf historische Entwicklungen zurückzuführen ist.

Erste Erfahrungen im Kontakt mit Geflüchteten machten die drei Gemeinden in den 1980er Jahren, als Menschen aus Polen sowie in den 1990er Jahren aus dem ehemaligen Jugoslawien als Asylwerber/innen nach Österreich kamen und in der Region vorübergehend wohnten. Diese Entwicklung betraf vor allem die Gemeinde A, jedoch zogen die meisten Menschen nach einiger Zeit wieder weg. Die Gemeinde B hatte seit den 1980er Jahren fast durchgehend Geflüchtete in Grundversorgungsquartieren aufgenommen. Die Gemeinde C ist mit der Integration von Geflüchteten hauptsächlich seit 2015 befasst. Zum Erhebungszeitpunkt im Jahr 2018 lebten nur noch wenige von ihnen in den Gemeinden A und C und vor allem kaum Familien, wie dies 2015 und 2016 der Fall war (Interview BürgERMEISTER GEMEINDE A 2018; Interview DiREKTOR NMS Gemeinde B 2018; Interview Amtsleiter Gemeinde C 2018). In den jeweiligen Orten waren allerdings bezogen auf die ehrenamtliche Unterstützung für Geflüchtete unterschiedliche Schwerpunkte festzustellen.

\section{„Willkommens-Verein“ in Gemeinde A}

Als im Herbst 2015 österreichweit Plätze zur Verteilung von Asylwerber/innen gesucht wurden, beschloss der Gemeinderat, jene Zahl an Asylwerber/innen aufzunehmen, die 2\% der Gemeindebevölkerung entsprach (Interview BürGERMEISTER GEMEINDE A 2018). Zeitgleich wurde von zivilgesellschaftlicher Seite ein Verein zur Unterstützung der Geflüchteten ins Leben gerufen, die in der Gemeinde (vorübergehend) ein Zuhause finden sollten. Der Gründung war eine Informationsveranstaltung von engagierten Gemeindebewohner/inne/n vorausgegangen, welche von circa 400 interessierten Bürger/inne/n besucht wurde. Diese Veranstaltung bot eine Möglichkeit, die Stimmungslage in der Gemeindebevölkerung sichtbar zu machen und auf dieser Basis weitere Schritte zu setzen. Da sich eine breite Unterstützungsbereitschaft seitens der Zivilgesellschaft abzeichnete, wurde der Verein schließlich gegründet, um das ehrenamtliche Engagement koordinieren zu können (Interview OBMANN „WiLlKommensVEREIN“ GEMEINDE A 2019). 
$\mathrm{Zu}$ Beginn, unmittelbar nach dem Eintreffen der ersten Geflüchteten im Ort, stand die Unterstützung durch Sachspenden im Vordergrund. Der „Willkommens-Verein“ eröffnete in einer Garage des örtlichen Rot-Kreuz-Hauses ein Sachspendenlager. Dieses Unterstützungsangebot wurde von Geflüchteten über die Gemeindegrenzen hinaus vor allem im Herbst und Winter 2015/16 sehr gut angenommen. Neben der finanziellen Unterstützung in diversen Belangen (es wurde ein Spendenkonto eingerichtet) und dem Sachspendenlager stellte vor allem der kostenlose Deutschunterricht eine wesentliche Säule dar. Dieser wurde über einen Zeitraum von drei Jahren von 30 bis 40 Ehrenamtlichen geleistet (ebd.).

Der „Willkommens-Verein“ hatte von der Gründung an eine starke Verbindung zur lokalen Pfarre. Beispielsweise wurden die Freiwilligen auch über das Pfarrblatt auf Möglichkeiten zur ehrenamtlichen Tätigkeit aufmerksam gemacht oder es wurden kulinarische Begegnungstreffen im Pfarrsaal organisiert. Der Verein wurde im Mai 2018 aufgelöst, da der Bedarf nicht mehr gegeben war. Dafür gibt es mehrere Gründe, die alle drei Gemeinden gleichermaßen betreffen: Zum einen hat sich gezeigt, dass sich geflüchtete Menschen nach Erhalt eines positiven Asylbescheids nicht langfristig in den Gemeinden angesiedelt haben. Als Asylberechtigte ziehen sie in größere Städte wie St. Pölten, sehr häufig nach Wien. Zum anderen lag der gesunkene Bedarf an Unterstützung auch daran, dass zum Erhebungszeitpunkt nur mehr wenige Asylwerber/innen neu in die Gemeinde kamen (ebd.).

\section{Pflichtschule in Gemeinde B}

In der Gemeinde B hatten seit den 1980er Jahren laufend Geflüchtete während der Zeit ihres Asylverfahrens Unterkunft in einem der beiden Grundversorgungsquartiere gefunden. In einer der Pensionen wurden hauptsächlich Familien mit Kindern untergebracht, weshalb die Direktor/inn/en und Lehrer/innen der örtlichen Volks- und Neuen Mittelschule viele Jahre Erfahrung hinsichtlich der Integration von geflüchteten Kindern haben. Im Jahr 2014 rief der Schulleiter der NMS schließlich das Projekt „Senior/inn/ en-Lehrer/inn/en“" ins Leben. Zum Erhebungszeitpunkt im Jahr 2018 engagierten sich circa zehn Pensionist/inn/en des Ortes ehrenamtlich für die Kinder, um mit ihnen in Kleinstgruppen spielerisch ihr Deutsch zu verbessern. Die Freiwilligenarbeit wurde von einer Lehrerin und der Schulleitung koordiniert; für die Ehrenamtlichen bedeuteten die Stunden mit den geflüchteten Kindern einen wöchentlichen Fixpunkt. Täglich wurde auf diese Art zweimal zwei Stunden pädagogische Arbeit geleistet - das entspricht 20 Wochenstunden und somit einer vollen Lehrverpflichtung (Interview DIREKTOR NMS GEMEINDE B 2018).

\section{Einzelpersonen in Gemeinde C}

In der Gemeinde C wurden seit 2015 ebenfalls Geflüchtete in einem dafür neu eingerichteten Grundversorgungsquartier aufgenommen. Auf Gemeindeseite wurde 
der Amtsleiter für die Geflüchteten zu einer wichtigen Ansprechperson. Er fungierte auch als Vermittler zwischen der Bevölkerung und den Asylwerber/inne/n, wenn es zum Beispiel um ehrenamtliche oder private Hilfstätigkeiten gegen geringe Entlohnung ging. In der Gemeinde $\mathrm{C}$ kristallisierte sich allerdings kein breites zivilgesellschaftliches Engagement heraus, wie dies in der Nachbargemeinde A der Fall war. Über die Diakonie gelangte schließlich im Herbst 2015 eine in Wien wohnhafte Ehrenamtliche in die Gemeinde, um mit den Geflüchteten integrativ-pädagogisch zu arbeiten. Dabei stand das Deutschlernen im Mittelpunkt, jedoch war sie darüber hinaus bemüht, Kontakte zwischen der Bevölkerung und den Geflüchteten zu knüpfen, weitere Einzelpersonen im Umfeld der Pfarre in ihren Deutschunterricht einzubinden und die Geflüchteten in Vereine und zu ehrenamtlicher Tätigkeit zu vermitteln. Der Deutschkurs fand einmal wöchentlich im Pfarrsaal statt und war zum Zeitpunkt der Erhebung eingestellt. Neben der ehrenamtlichen Unterstützerin aus Wien waren engagierte Einzelpersonen aktiv, allerdings keine Organisation oder ein Verein wie in der Nachbargemeinde A (Interview AmtSLEITER Gemeinde C 2018; Interview EhrenAmtLiche UnterstütZerin Gemeinde C 2018).

\subsection{Fazit}

Die Beschreibungen der beiden Erhebungsgebiete zeigen, dass der ländliche Raum sehr unterschiedliche Voraussetzungen für die Integration von Geflüchteten bietet. An dieser Stelle muss erwähnt werden, dass diese Regionen nach unterschiedlichen Kriterien ausgewählt wurden und daher im Forschungsansatz keine von vornherein vergleichende Perspektive angelegt war. Dennoch scheint ein komparativer Blick auf gewisse Aspekte aufschlussreich zu sein, um sich mit der Integration von Geflüchteten im ländlichen Raum genauer auseinanderzusetzen. In beiden Regionen spielte die Zivilgesellschaft bei der lokalen Integration eine bedeutende Rolle. In allen untersuchten Gemeinden existierten Unterstützungsangebote für Geflüchtete, wenngleich diese recht unterschiedlich ausgestaltet waren. In St. Andrä-Wördern sowie in der Gemeinde A wurde ehrenamtliches Engagement durch Vereine organisiert. Damit konnten beispielsweise freiwilliger Deutschunterricht koordiniert sowie Sach- und Geldspenden verwaltet werden. Zivilgesellschaftliche Vereine bemühen sich meist auch um die Organisation von Veranstaltungen mit dem Ziel Begegnungsräume für die Gemeindebevölkerung zu schaffen, welche auch für Geflüchtete offenstehen. In der Gemeinde C wurde zivilgesellschaftliches Engagement hingegen von Einzelpersonen geleistet und in Gemeinde B fokussierte sich ehrenamtliches Engagement auf Kinder und Jugendliche in der örtlichen Pflichtschule. Generell zeigt sich, dass die lokalen Umstände eine wichtige Rolle spielen und auf die Verwirklichungschancen von Geflüchteten in den Gemeinden großen Einfluss haben. 